Ying Xiong* and Chuan Gao

\title{
Crystal structure of $N^{\prime}, N^{\prime \prime \prime}-$-((1E, $\left.1^{\prime} E\right)$-((decane- 1,10-diylbis(oxy))bis(2,1-phenylene)) bis(methaneylylidene))di(isonicotinohydrazide), $\mathrm{C}_{36} \mathrm{H}_{40} \mathrm{~N}_{6} \mathrm{O}_{4}$
}

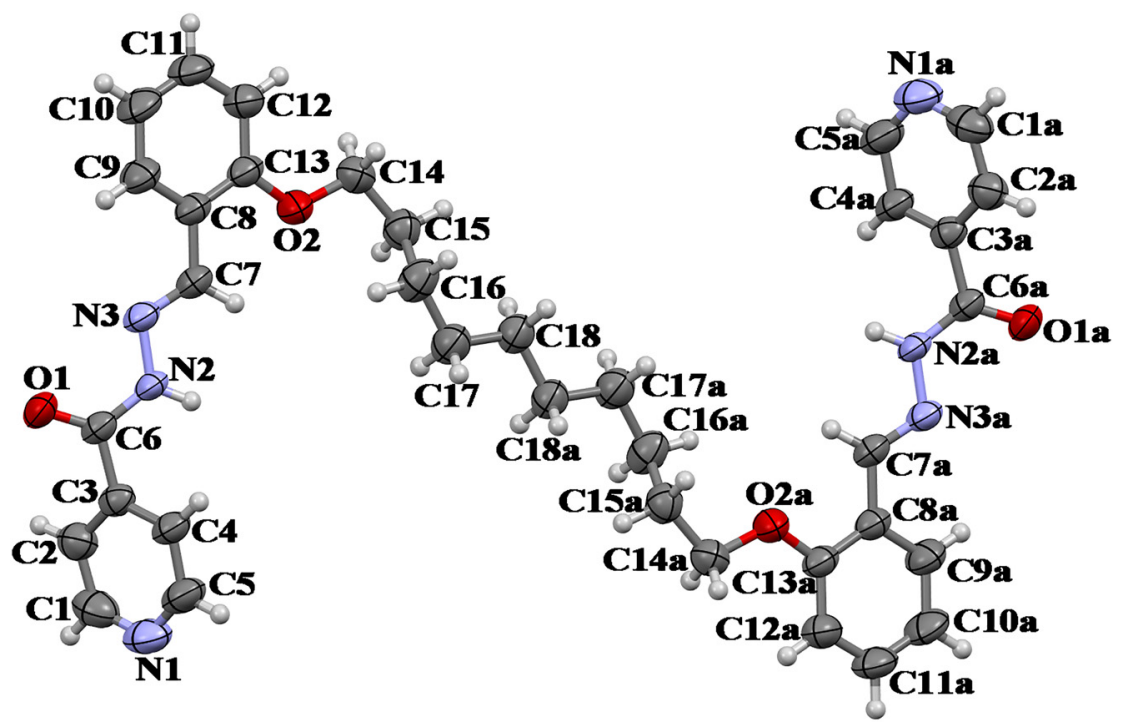

https://doi.org/10.1515/ncrs-2021-0125

Received April 6, 2021; accepted April 26, 2021; published online May 11, 2021

\section{Abstract}

$\mathrm{C}_{36} \mathrm{H}_{40} \mathrm{~N}_{6} \mathrm{O}_{4}$, monoclinic, $P 2_{1} / c$ (no. 14), $a=11.094(6) \AA$, $b=17.694(11) \AA, \quad c=8.698(5) \AA, \quad \beta=103.56(2)^{\circ}$, $V=1659.9(16) \AA^{3}, Z=2, R_{\mathrm{gt}}(F)=0.0470, w R_{\mathrm{ref}}\left(F^{2}\right)=0.1375$, $T=273 \mathrm{~K}$.

CCDC no.: 2060577

The molecular structure is shown in the figure. Table 1 contains crystallographic data and Table 2 contains the list of the atoms including atomic coordinates and displacement parameters.

*Corresponding author: Ying Xiong, School of Chemistry and Materials, Guizhou Normal University, Guiyang, 550025, People’s Republic of China, E-mail: 124251038@qq.com. https://orcid.org/ 0000-0002-5790-3396

Chuan Gao, School of Chemistry and Materials, Guizhou Normal University, Guiyang, 550025, People's Republic of China
Table 1: Data collection and handling.

\begin{tabular}{ll}
\hline Crystal: & Colourless block \\
Size: & $0.25 \times 0.22 \times 0.20 \mathrm{~mm}$ \\
Wavelength: & Mo $K \alpha$ radiation $(0.71073 \AA)$ \\
$\mu:$ & $0.08 \mathrm{~mm}^{-1}$ \\
Diffractometer, scan mode: & $\mathrm{D} 8 / \mathrm{APEX} 2, \varphi$ and $\omega$ \\
$\theta_{\text {max }}$, completeness: & $25.0^{\circ},>99 \%$ \\
$N(h k l)_{\text {measured }}, N(h k l)_{\text {unique }}, R_{\text {int }}:$ & $30844,2926,0.081$ \\
Criterion for $I_{\text {obs }}, N(h k l)_{\text {gt }}:$ & $I_{\text {obs }}>2 \sigma\left(I_{\text {obs }}\right), 1983$ \\
$N(\text { param })_{\text {refined }}:$ & 208 \\
Programs: & Olex2 [1], Bruker [2], SHELX [3], \\
& Diamond [4] \\
\hline
\end{tabular}

\section{Source of material}

A solution of 2,2'-(decane-1,10-diylbis(oxy))dibenzaldehyde $(0.76 \mathrm{~g}, 2.0 \mathrm{mmol})$ in ethanol $(50 \mathrm{~mL})$ was added to a stirred ethanol solution $(50 \mathrm{~mL})$ of isonicotinohydrazide $(0.55 \mathrm{~g}, 4.0 \mathrm{mmol})$. The reaction mixture was refluxed for $8 \mathrm{~h}$, then the mixture was cooled to room temperature. The white precipitated residue was removed by filtration and then washed with ethanol three times. The white solids 
Table 2: Fractional atomic coordinates and isotropic or equivalent isotropic displacement parameters $\left(* / \AA^{2}\right)$.

\begin{tabular}{|c|c|c|c|c|}
\hline Atom & $x$ & $y$ & $z$ & $U_{\text {iso }}{ }^{*} / U_{\text {eq }}$ \\
\hline $\mathrm{C} 1$ & $0.0084(2)$ & $0.16008(15)$ & $0.1557(4)$ & $0.0806(8)$ \\
\hline $\mathrm{H} 1$ & -0.071239 & 0.163079 & 0.091178 & $0.097^{*}$ \\
\hline $\mathrm{C} 2$ & $0.1064(2)$ & $0.18228(13)$ & $0.0925(3)$ & $0.0678(6)$ \\
\hline $\mathrm{H} 2 \mathrm{~A}$ & 0.092771 & 0.197627 & -0.012384 & $0.081^{*}$ \\
\hline C3 & 0.22485 (19) & $0.18116(11)$ & $0.1887(2)$ & $0.0516(5)$ \\
\hline $\mathrm{C} 4$ & $0.2390(2)$ & $0.15567(12)$ & $0.3411(3)$ & $0.0600(6)$ \\
\hline H4 & 0.317066 & 0.153934 & 0.409752 & $0.072^{*}$ \\
\hline C5 & $0.1352(3)$ & $0.13264(14)$ & $0.3908(3)$ & $0.0776(7)$ \\
\hline H5 & 0.146514 & 0.114368 & 0.493395 & $0.093^{*}$ \\
\hline C6 & 0.33254 (19) & $0.20559(11)$ & $0.1242(2)$ & $0.0512(5)$ \\
\hline $\mathrm{C7}$ & 0.58877 (19) & $0.31823(12)$ & $0.2782(2)$ & $0.0532(5)$ \\
\hline $\mathrm{H} 7$ & 0.564944 & 0.330439 & 0.370833 & $0.064^{*}$ \\
\hline C8 & $0.70311(18)$ & $0.35032(12)$ & $0.2499(2)$ & $0.0537(5)$ \\
\hline C9 & $0.7602(2)$ & $0.32037(14)$ & $0.1379(3)$ & $0.0697(7)$ \\
\hline H9 & 0.726954 & 0.277761 & 0.080786 & $0.084^{*}$ \\
\hline $\mathrm{C} 10$ & $0.8667(2)$ & $0.35349(17)$ & $0.1101(3)$ & $0.0820(8)$ \\
\hline $\mathrm{H} 10$ & 0.904385 & 0.333250 & 0.034448 & $0.098^{*}$ \\
\hline C11 & $0.9159(2)$ & $0.41611(17)$ & $0.1946(3)$ & $0.0785(7)$ \\
\hline H11 & 0.986951 & 0.438267 & 0.175326 & $0.094^{*}$ \\
\hline C12 & $0.8623(2)$ & $0.44654(14)$ & $0.3070(3)$ & $0.0696(6)$ \\
\hline $\mathrm{H} 12$ & 0.896764 & 0.488946 & 0.363811 & $0.084^{*}$ \\
\hline C13 & $0.75605(18)$ & $0.41364(13)$ & $0.3356(2)$ & $0.0562(5)$ \\
\hline C14 & $0.7509(2)$ & $0.49630(13)$ & $0.5546(3)$ & $0.0657(6)$ \\
\hline $\mathrm{H} 14 \mathrm{~A}$ & 0.760950 & 0.543301 & 0.501521 & $0.079 *$ \\
\hline H14B & 0.831819 & 0.479380 & 0.613280 & 0.079 * \\
\hline C15 & $0.6654(2)$ & $0.50723(13)$ & $0.6638(3)$ & $0.0664(6)$ \\
\hline $\mathrm{H} 15 \mathrm{~A}$ & 0.584805 & 0.523297 & 0.602462 & $0.080^{*}$ \\
\hline $\mathrm{H} 15 \mathrm{~B}$ & 0.697894 & 0.547081 & 0.738637 & $0.080^{*}$ \\
\hline C16 & $0.6498(3)$ & $0.43612(13)$ & $0.7540(3)$ & $0.0762(7)$ \\
\hline $\mathrm{H} 16 \mathrm{~A}$ & 0.726171 & 0.426769 & 0.832536 & $0.091^{*}$ \\
\hline H16B & 0.636621 & 0.393841 & 0.680962 & $0.091^{*}$ \\
\hline C17 & $0.5431(2)$ & $0.43894(14)$ & $0.8367(3)$ & $0.0741(7)$ \\
\hline $\mathrm{H} 17 \mathrm{~A}$ & 0.466142 & 0.444668 & 0.757177 & $0.089^{*}$ \\
\hline H17B & 0.539445 & 0.390976 & 0.889436 & $0.089^{*}$ \\
\hline C18 & $0.5515(2)$ & 0.50107 (13) & $0.9557(3)$ & $0.0657(6)$ \\
\hline $\mathrm{H} 18 \mathrm{~A}$ & 0.548821 & 0.549311 & 0.902034 & $0.079 *$ \\
\hline H18B & 0.630832 & 0.497592 & 1.031572 & 0.079 * \\
\hline N1 & $0.0205(2)$ & $0.13490(13)$ & $0.3016(3)$ & $0.0849(7)$ \\
\hline N2 & $0.41619(15)$ & $0.24825(9)$ & $0.22356(19)$ & $0.0526(4)$ \\
\hline $\mathrm{H} 2$ & 0.404775 & 0.259711 & 0.315175 & $0.063^{*}$ \\
\hline N3 & $0.52103(15)$ & $0.27385(10)$ & $0.1786(2)$ & 0.0544 (4) \\
\hline 01 & $0.33980(14)$ & $0.18754(8)$ & $-0.00953(17)$ & $0.0646(4)$ \\
\hline 02 & 0.69441 (14) & $0.44041(9)$ & $0.44244(18)$ & $0.0718(5)$ \\
\hline
\end{tabular}

$(0.1 \mathrm{mmol})$ were dissolved in a solution mixture $(20.0 \mathrm{~mL}$, $\left.\mathrm{CH}_{3} \mathrm{OH} / \mathrm{DMF}, 9: 1, v / v\right)$ within three days. Crystals were obtained by slow evaporation at room temperature.

\section{Experimental details}

Using Olex2 [1], the structure was solved using Charge Flipping and refined with the ShelXL [3] refinement. All hydrogen atoms were positioned geometrically, with the $d(\mathrm{C}-\mathrm{H})=0.97-0.99 \AA, U_{\text {iso }}(\mathrm{H})=1.2$ times $U_{\text {eq }}$ (parent atom).

\section{Comment}

A bis-pyridylhydrazone Schiff base ligand was synthesized. The nitrogen atoms on the pyridine ring have strong nucleophilic ability [5]. Acylhydrazone Schiff base compounds can be easily assembled with different transition metal ions in different coordination modes to form novel complexes [6].

In the crystal structure of the title compound, the asymmetric unit of the title compound consists of one target molecule. The title molecule shows a slightly twisted " $Z$ " shape (see the Figure). The bond lengths and bond angles are in the normal ranges [6]. The amide forms an intermolecular hydrogen bond with the amide $\mathrm{N}$ on another molecule. The intermolecular $\mathrm{N}-\mathrm{H} \cdots \mathrm{O}$ hydrogen bonds in the target compound extend the framework into a $2 D$ structure.

Author contributions: All the authors have accepted responsibility for the entire content of this submitted manuscript and approved submission.

Research funding: This work was supported by the Science and Technology Foundation of Guizhou Province (grant number [2019]2792).

Conflict of interest statement: The authors declare no conflicts of interest regarding this article.

\section{References}

1. Dolomanov O. V., Bourhis L. J., Gildea R. J., Howard J. A. K., Puschmann H. OLEX2: a complete structure solution, refinement and analysis program. J. Appl. Crystallogr. 2009, 42, 339-341.

2. Bruker. APEX2, SAINT and SADABS; Bruker AXS Inc.: Madison, Wisconsin, USA, 2012.

3. Sheldrick G. M. Crystal structure refinement with SHELXL. Acta Crystallogr. 2015, C71, 3-8.

4. Brandenburg K. DIAMOND. Visual Crystal Structure Information System. Ver. 4.0; Crystal Impact: Bonn, Germany, 2015.

5. Zhang N., Yuan J., Liu M. J. Synthesis and magnetism of binuclear fe complexes based on bis-pyridylhydrazone Schiff base ligands. Chin. J. Inorg. Chem. 2019, 11, 2066-2072.

6. Hai S. K., Lou S. F., Qiu X. Y. Syntheses, crystal structures and antimicrobial activity of copper and zinc complexes with aroylhydrazones. Chin. J. Inorg. Chem. 2016, 32, 906-912. 